\title{
Article \\ Rough Set Based Classification and Feature Selection Using Improved Harmony Search for Peptide Analysis and Prediction of Anti-HIV-1 Activities
}

\author{
Bagyamathi Mathiyazhagan ${ }^{1}$, Joseph Liyaskar ${ }^{2}$, Ahmad Taher Azar ${ }^{3,4, * \mathbb{D}}$, Hannah H. Inbarani ${ }^{5}$, Yasir Javed ${ }^{3} \mathbb{D}$, \\ Nashwa Ahmad Kamal ${ }^{6}$ and Khaled M. Fouad ${ }^{4,7}$
}

Citation: Mathiyazhagan, B.; Liyaskar, J.; Azar, A.T.; Inbarani, H.H.; Javed, Y.; Kamal, N.A.; Fouad, K.M. Rough Set Based Classification and Feature Selection Using Improved Harmony Search for Peptide Analysis and Prediction of Anti-HIV-1 Activities. Appl. Sci. 2022, 12, 2020. https://doi.org/10.3390/ app12042020

Academic Editors: Hélder P. Oliveira, António Cunha and Tania Pereira

Received: 14 January 2022

Accepted: 9 February 2022

Published: 15 February 2022

Publisher's Note: MDPI stays neutral with regard to jurisdictional claims in published maps and institutional affiliations.

Copyright: (C) 2022 by the authors. Licensee MDPI, Basel, Switzerland. This article is an open access article distributed under the terms and conditions of the Creative Commons Attribution (CC BY) license (https:// creativecommons.org/licenses/by/ $4.0 /)$.
1 Gonzaga College of Arts and Science for Women, Krishnagiri 635108, India; bagyaarul@gmail.com

2 Government Mohan Kumaramangalam Medical College and Hospital, Salem 636030, India; jliyaskar@gmail.com

3 College of Computer and Information Sciences, Prince Sultan University, Riyadh 11586, Saudi Arabia; yjaved@psu.edu.sa

4 Faculty of Computers and Artificial Intelligence, Benha University, Benha 13518, Egypt; kmfi@fci.bu.edu.eg or kmo-hamed@nu.edu.eg

5 Department of Computer Science, Periyar University, Salem 636011, India; hhinba@gmail.com

6 Faculty of Engineering, Cairo University, Giza 12613, Egypt; nashwa.ahmad.kamal@gmail.com

7 Faculty of Information Technology and Computer Science, Nile University, Shikh Zaid 12568, Egypt

* Correspondence: aazar@psu.edu.sa or ahmad.azar@fci.bu.edu.eg or ahmad_t_azar@ieee.org
Abstract: AIDS, which is caused by the most widespread HIV-1 virus, attacks the immune system of the human body, and despite the incredible endeavors for finding proficient medication strategies, the continuing spread of AIDS and claiming subsequent infections has not yet been decreased. Consequently, the discovery of innovative medicinal methodologies is highly in demand. Some available therapies, based on peptides, proclaim the treatment for several deadly diseases such as AIDS and cancer. Since many experimental types of research are restricted by the analysis period and expenses, computational methods overcome the issues effectually. In computational technique, the peptide residues with anti-HIV-1 activity are predicted by classification method, and the learning process of the classification is improved with significant features. Rough set-based algorithms are capable of dealing with the gaps and imperfections present in real-time data. In this work, feature selection using Rough Set Improved Harmony Search Quick Reduct and Rough Set Improved Harmony Search Relative Reduct with Rough Set Classification framework is implemented to classify Anti-HIV-1 peptides. The primary objective of the proposed methodology is to predict the peptides with an anti-HIV-1 activity using effective feature selection and classification algorithms incorporated in the proposed framework. The results of the proposed algorithms are comparatively studied with existing rough set feature selection algorithms and benchmark classifiers, and the reliability of the algorithms implemented in the proposed framework is measured by validity measures, such as Precision, Recall, F-measure, Kulczynski Index, and Fowlkes-Mallows Index. The final results show that the proposed framework analyzed and classified the peptides with a high predictive accuracy of $96 \%$. In this study, we have investigated the ability of a rough set-based framework with sequencebased numeric features to classify anti-HIV-1 peptides, and the experimentation results show that the proposed framework discloses the most satisfactory solutions, where it rapidly congregates in the problem space and finds the best reduct, which improves the prediction accuracy of the given dataset.

Keywords: rough set theory; feature selection; improved harmony search; anti-HIV-1 peptides; peptide therapeutics; rough set classification

\section{Introduction}

AIDS (Acquired Immunodeficiency Syndrome) is a deadly and overwhelming disease caused by the HIV virus [1]. According to the World Health Organization (WHO) survey, 
more than 35 million people have died, and 36.9 million live with HIV globally. The HIV-1 type virus is the most widespread type and major stimulator of AIDS [2]. Many therapeutics have been discovered to save the infected person, but recovery is a challenging process; therefore, innovative and effective treatment for AIDS is needed. Long-term medication and toxicity may restrict the utilization of such treatments, and it should be frequently monitored and controlled by another supportive therapy [3]. Peptide molecules are created by the dehydration condensation reaction of amino acids joined by peptide bonds. They are used to treat a variety of diseases [4]. In recent years, peptides that have an anti-HIV-1 activity are being focused on and reveal promising results, which gradually reduce the effects of AIDS [5]. Investigating the enormous anti-HIV-1 peptides demands much time and effort, where simplified methodologies, such as machine learning, can be developed and implemented to predict the anti-HIV-1 peptides [6].

Feature Selection (FS) is one of the significant phases in the proposed framework, which eliminates redundant and irrelevant features to increase classification accuracy [7]. The prediction of anti-HIV-1 peptides (AHP) from a given sequence data would be well improvised, as it decreases the running time of the classification phase. The Harmony Search (HS) algorithm is based on a music enhancing process that searches for an optimal harmony [8-11]. The HS algorithm is modified by Mahdavi et al. [12,13] in terms of parameters. Rough Set theory is a tool to deal with the ambiguity and redundancy present in the dataset and reserves its originality [14]. The feature selection and classification techniques using rough set theory are effectively implemented in many studies [15,16]. An improved HS is embedded with RST for attribute selection to analyze protein sequences [17].

In the proposed framework, the essence of Rough Set Quick Reduct and Rough Set Relative Reduct with an improved HS algorithm is employed to select the significant features. A Rough Set Classification (RSC) is employed to form the rules for the selected features and predict anti-HIV-1 peptides. The RSC algorithm classifies the significant features, and the performance of the Rough Set Particle Swarm Optimization Quick Reduct (RSPSOQR) and Rough Set Particle Swarm Optimization Relative Reduct (RSPSORR) feature selection algorithms are comparatively analyzed with existing Particle Swarm Optimization (PSO) based algorithms. The accuracy of the RSC algorithm is evaluated with benchmark classifiers.

\section{Research Motivation and Contribution}

HIV is a deadly disease that should be treated with more effective therapy, and peptide-based treatment that fights against the virus has proved beneficial compared to other treatments. The conventional experiments on these peptides, as known, require much time and effort, where the peptide with AHP activity can be determined by computational intelligence techniques [6]. Generally, the dataset extracted from the biological sequences is large in dimension, with inconsistencies, where the dimensionality reduction method to handle the inconsistency in the peptide dataset is a requirement. The RSC algorithm is adopted in the proposed framework along with Rough Set Improved Harmony Search Quick Reduct (RSIHSQR) and Rough Set Improved Harmony Search Relative Reduct (RSIHSRR) feature selection algorithm. In Section 3 Figure 1 shows the proposed framework that depicts and predicts the anti-HIV-1 peptides with a good accuracy using finest selected features. As the RSIHSQR and RSIHSRR feature selection algorithm has revealed the best result in the previous studies [17], it is also employed for the peptide therapeutic field of this study. 


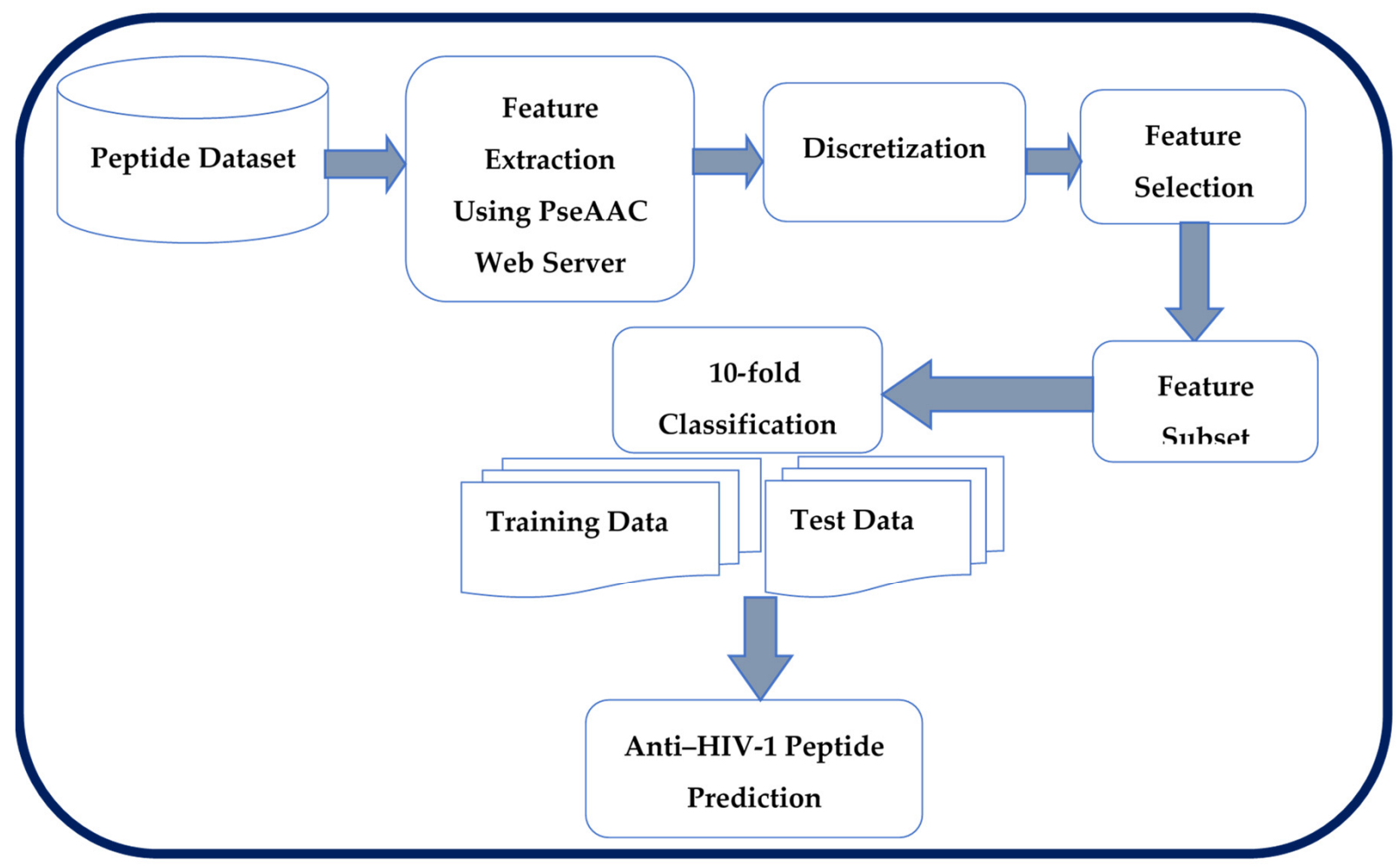

Figure 1. The Proposed Framework.

The rest of the paper is organized as follows: Section 2 presents the related work of this study. Section 3 presents the proposed methods for Peptide Classification. Details about RSC are presented in Section 4. Experimental analysis and results are presented in Section 5. Discussion is presented in Section 6. Conclusions with future work are presented in Section 7.

\section{Related Work}

The implementation of the machine learning method in bioinformatics is affected by many factors. The peptide therapeutic field describes different types of frameworks using the computational method. It consumes enormous effort, time, and cost to analyze peptide information. The knowledge discovery for the bioinformatics applications will be a challenging process due to a large-sized database. The framework that embeds the feature selection before classification effectively handles the high-dimensional proteomic datasets. In the last two decades, significant research endeavors have been committed with data mining systems to acquire information in the field of disease diagnosis [15,18-20]. As accuracy is most important for diagnosing diseases and treating patients, the Rough Set is one such tool to deal with uncertain and imprecise data and yield accurate results. There are considerable numbers of studies on feature selection techniques, and classification methods are depicted in Table 1.

Table 1. Related work of this study.

\begin{tabular}{cl}
\hline \multicolumn{1}{c}{ Author } & \multicolumn{1}{c}{ Purpose and Methodology } \\
\hline & $\begin{array}{l}\text { Anti-HIV-1 peptides are predicted using the } \\
\text { InfoGainAttributeEval feature selection } \\
\text { algorithm and MLP, K-Star, J48, Random } \\
\text { Poorinmohammad et al. [6] } \\
\end{array}$ \\
& $\begin{array}{l}\text { Forest, and LMT classification algorithms on } \\
\text { the Weka framework. }\end{array}$ \\
\hline
\end{tabular}


Table 1. Cont. Related work of this study.

\begin{tabular}{l} 
Author \\
\hline Bagyamathi and Inbarani [7] \\
Inbarani et al. [21]
\end{tabular}

Bagyamathi and Inbarani [22]

Meher eta 1. [23]

The protein sequences are classified into four structural classes using benchmark classifiers. The RSIHSQR feature selection algorithm gradually improves the precision of the classification algorithm.

The primary protein sequences are classified with the PseAAC feature subset selected by Rough Set Black Hole Quick Reduct (RSBHQR) and Rough Set Black Hole Relative Reduct (RSBHRR) benchmark classification algorithms. In this study, the structural protein classes are predicted by RSC algorithm using the selected features by the RSIHSQR algorithm.

In this study, anti-microbial peptides are predicted with a support vector machine (SVM) using physicochemical and structural features extracted from peptides and developed an aiAMPpred online tool for the prediction of the anti-microbial peptide.

Azar et al. [24]

In this study, the Pessimistic Multi-Granulation Rough Sets (PMGRS) classification algorithm is implemented to diagnose heart valve disease. In this study, the antiviral peptides are

Zare et al. [25] predicted by RBF, Naïve Bayes, J48, Decision Stump, and REPTree classification techniques. In this study, the imperative features are

Bagyamathi and Inbarani [26] selected by RSIHSRR algorithm for the medical data classification.

The structural classes of the protein are predicted using the subset of features selected by RSIHSRR algorithm, and classification

Bagyamathi and Inbarani [27] algorithms evaluate the originality of the feature subset.

In this work, the peptides are modeled by statistical methods by concurrently predicting

Barrett et al. [28] amino acid sequence motifs. The motif-based method is used to elucidate the functional motifs in anti-microbial activity.

This work investigates principal component

Tantisatirapong et al. [29] analysis, max-relevance, and min-redundancy feed-forward selection for brain tumor classification.

This study uses a SVM classification algorithm

Hajisharifi et al. [30] on anti-cancer. Chou's PseAAC based features are applied to the proposed classification algorithm.

Inbarani et al. [31]

This study analyzes the medical dataset with RSPSOQR and RSPSORR feature selection for disease identification.

This study proposes a linguistic hedges

Azar et al. [32] neuro-fuzzy classifier (LHNFCSF) for the medical diagnosis with the selected features. In this paper, the mammogram images are

Jothi et al. [33] selected using STRSPSOQR and STRSPSO-RR algorithms to select the best features. 


\section{Proposed Method for Peptide Classification}

The proposed methodology shown in Figure 1 collects the peptides with AHP from the HIV inhibitory peptides (HIPdb) database [34]. Physicochemical properties based on features are extracted from the peptides using Chou's PseAAC method [20]. The extracted peptide dataset is discretized for the rough set-based analysis. It is subjected to a feature selection process to reveal the relevant features and classification algorithm used for peptides prediction. This section discusses the major components of the proposed framework.

\subsection{Feature Extraction}

Feature extraction is used in the proposed framework to convert sequences to numerical attributes, as shown in Figure 2. The peptides are converted to digital vector using PseAAC server for the computational process [20,35], where the peptide features are extracted from conventional AAC with six physicochemical properties with weight factor (0.05) and lambda given the value of 2, where the 6 physicochemical properties are: Hydrophobicity, Hydrophilicity, pK1 (Ca-COOH), pK2 (NH3), PI (25 _C), and molecular weight. The 63 combinations of physicochemical properties are considered, and 126 features are extracted $(\lambda=2)$ from the peptide dataset for sequence order information [36]. These features are generated from type 1 of the PseAAC web server (http:/ / www.csbio.sjtu.edu.cn/bioinf/PseAAC, accessed on 10 January 2022).

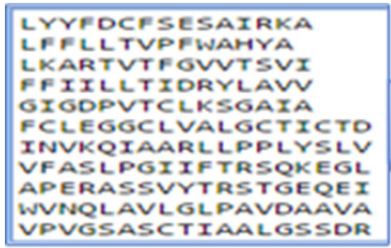

\begin{tabular}{|c|c|c|c|c|c|c|c|c|c|c|c|}
\hline & 9.007 & 4.446 & 8.18 & 6.272 & 9.066 & 7.1 & 4.78 & 6.671 & 5.695 & 7.131 & 6.78: \\
\hline & 9.33 & 12.47 & 3.958 & 7.478 & 8.907 & 10.94 & 12.482 & 8.424 & 10.056 & 10.737 & 10.928 \\
\hline & 10.637 & 11.478 & 11.884 & 6.396 & 7.253 & 8.797 & 9.237 & 9.451 & 6.638 & 7.068 & 11.00 \\
\hline \multirow{6}{*}{ PseAAC Web } & 12.352 & 12.73 & 9.674 & 13.115 & 18.743 & 14.653 & 14.832 & 13.403 & 15.016 & 17.801 & $12.54:$ \\
\hline & 12.352 & 12.62 & 6.683 & 14.28 & 19.105 & 15.278 & 15.404 & 12.715 & 16.189 & 18.564 & 12.486 \\
\hline & 9.325 & 5.702 & 7.22 & 8.353 & 9.919 & 9.352 & 7.577 & 8.312 & 8.868 & 9.649 & 7.545 \\
\hline & 6.273 & 7.263 & 18.366 & 12.542 & 5.433 & 5.441 & 5.947 & 12.015 & 8.74 & 5.015 & 6.77 \\
\hline & 6.583 & 8.512 & 15.325 & 9.082 & 3.053 & 5.528 & 6.524 & 10.215 & 6.823 & 3.756 & 7.558 \\
\hline & 5.426 & 10.315 & 2.366 & 7.083 & 5.605 & 7.927 & 10.307 & 6.5 & 8.719 & 8.011 & 7.938 \\
\hline
\end{tabular}

Figure 2. Features extracted from anti-HIV-1 peptide sequence.

\subsection{Discretization of Peptide Features}

The uncertain dataset with real-valued attributes is discretized for an effective classification $[37,38]$. Discretization is the process of transferring continuous values to discrete ones, and rough set theory works on discrete variables to deal with the ambiguity presented in the real dataset [39]. The peptide dataset experimented with within this study has realvalued attributes. Hence, it is discretized using the Max-Min discretization method [40,41], then it was experimented with by the feature selection algorithms in the proposed framework.

\subsection{Rough Set Based Feature Selection}

Feature selection (FS) is a dimension reduction method for datasets with many attributes, where the distinctiveness of the dataset is preserved [2]. The rough set selects the relevant features based on the FS algorithms, which improves the learning process of prediction methods [18,42]. In this work, RSIHSQR and RSIHSRR feature selection algorithms are implemented to diminish the redundant and immaterialized features of the available datasets and disclose the best features. Both algorithms are implemented for a large dataset and have shown likely results in protein analysis [22,43]. The spirit of the rough set quick reduct method, in the proposed algorithms, is inherited in the improved HS algorithm to converge towards the best solution, and the solution is achieved by the music improvisation technique $[13,44]$, where both algorithms are briefly described in $[17,26]$. The results of Algorithm 1 and Algorithm 2 are relatively studied with RSPSOQR and RSPSORR algorithms [33,45], and the selected best features are used as an input to the classifier for the analysis and classification of anti-HIV-1 peptides. 


\section{Algorithm 1. RSIHSQR FS algorithm \\ Algorithm: RSIHSQR(C,De) \\ Input: $C$, the conditional attributes; \\ De, the class attribute; \\ Output: Optimal feature subset}

Step I: The fitness function, $f(X)$ to be defined.

Initialize the parameters HMS $=30$
$H M C R=0.9$
// HM constraint
MaxIt $=100$
// iteration count

$P V B / /$ feasible value limit

$P A R_{\min }, P A R_{\max }, b w_{\min }, b w_{\max }, / /$ Pitch Adjusting Rate \& bandwidth $\in(0$ to 1)

fit $=0$;

$X_{\text {old }}=X_{1} ;$ bestfit $=X_{1}$; bestreduct $=\{\}$

Step II: The Harmony Memory to be set as, $H M=\left(X_{1}, X_{2}, \ldots, X_{H M S}\right)$

For $i=1: H M S$

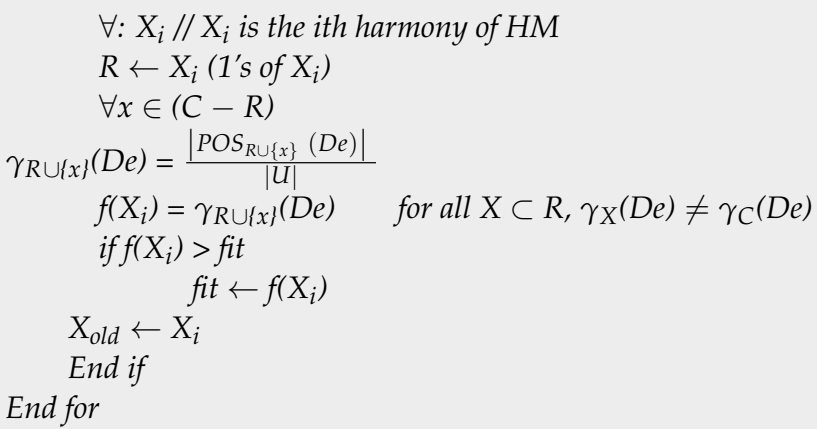

Step III: The new HM to be improvised.

While it $\leq$ MaxIt $\mid$ fit $==1$

for $j=1: N V A R$

Update PAR();

$\forall: X_{\text {old }}(j)$

Update bw();

if random ( ) $\leq$ HMCR $\quad / /$ random numberbetween 0 and 1

$X_{\text {new }} \leftarrow X_{\text {old }}$

$$
\begin{gathered}
\text { if random }() \leq P A R \\
X_{\text {new }}(j)=X_{\text {new }}(j) \pm \operatorname{random}() * b w \\
\text { end if }
\end{gathered}
$$

else

// select Xnew

end if

$$
X_{\text {new }}(j)=P V B_{\text {lower }}+\operatorname{random}() *\left(P V B_{\text {upper }}-P V B_{\text {lower }}\right)
$$

end for

Step IV: The new HM to be updated

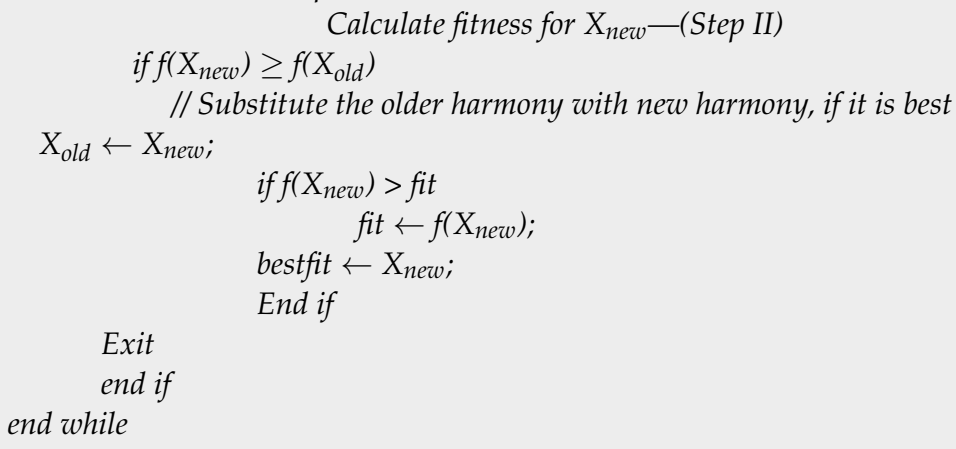




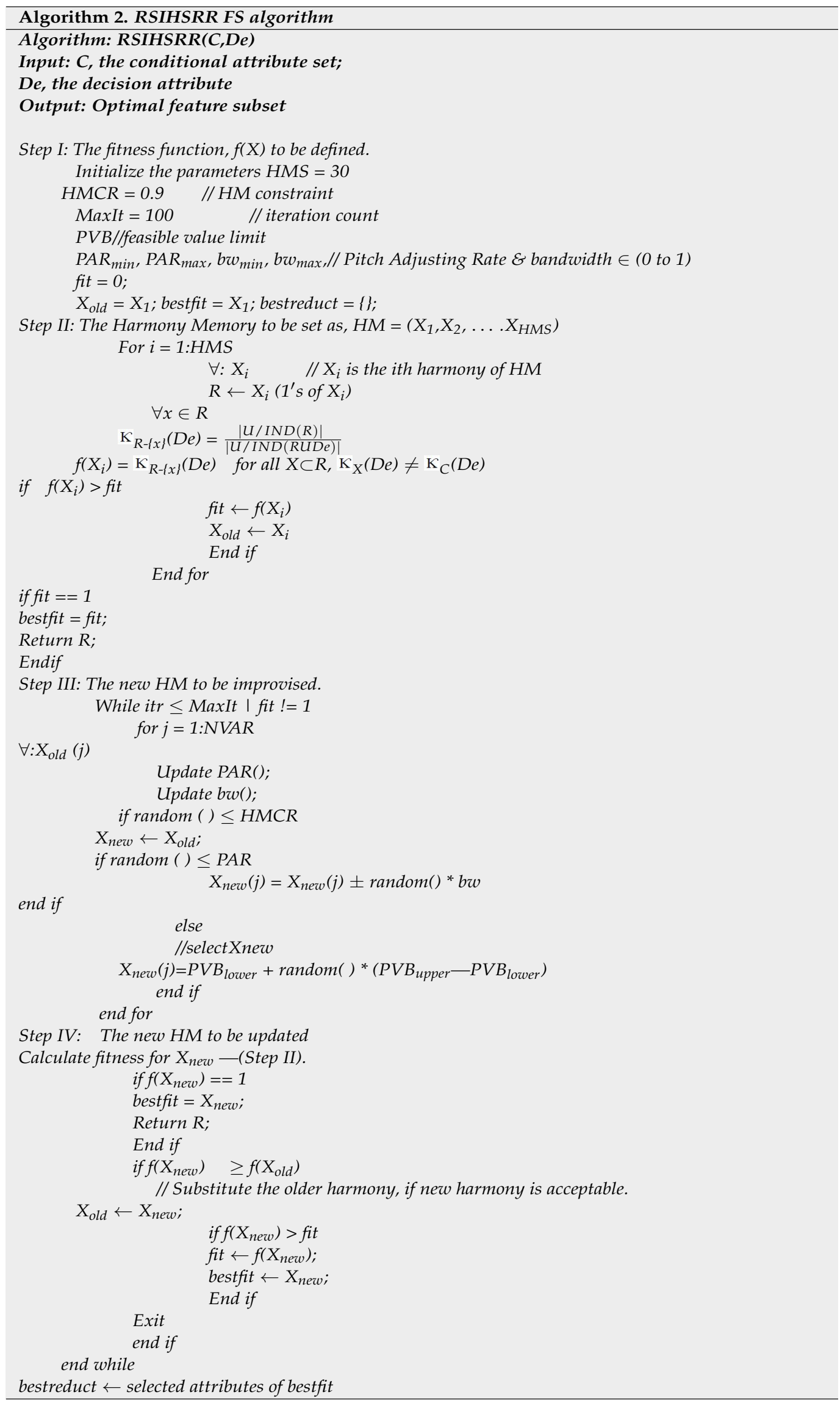




\section{Rough Set Classification (RSC)}

Classification is another vital method for discovering knowledge in bioinformatics applications [7]. In the proposed framework, the peptide dataset is classified using a Rough RSC algorithm based on the following objectives [46,47]: (a) RST is simple to implement, and it can be extended with any algorithms; (b) it does not require any input parameter except dataset; (c) it delivers better analysis result for the knowledge-making process; (d) it withstands uncertainties and imperfections present in the dataset. It works on a 10-fold cross-validation method to produce the decision rules and the lower and upper approximation of the RST. The equivalence relation of conditional and decision attributes classifies the peptides into two disjoint classes: anti-HIV-1 activity and low/nil anti-HIV-1 activity. The peptides are classified to forecast the anti-HIV-1 activity based on the constructed rules. The functioning of the RSC algorithm is comparatively assessed with five benchmark classifiers [48] in terms of Precision, Recall, F-Measure, Kulczynski Index, and Fowlkes-Mallows Index [49]. The proposed RSC algorithm is represented in Algorithm 3 in the following table.

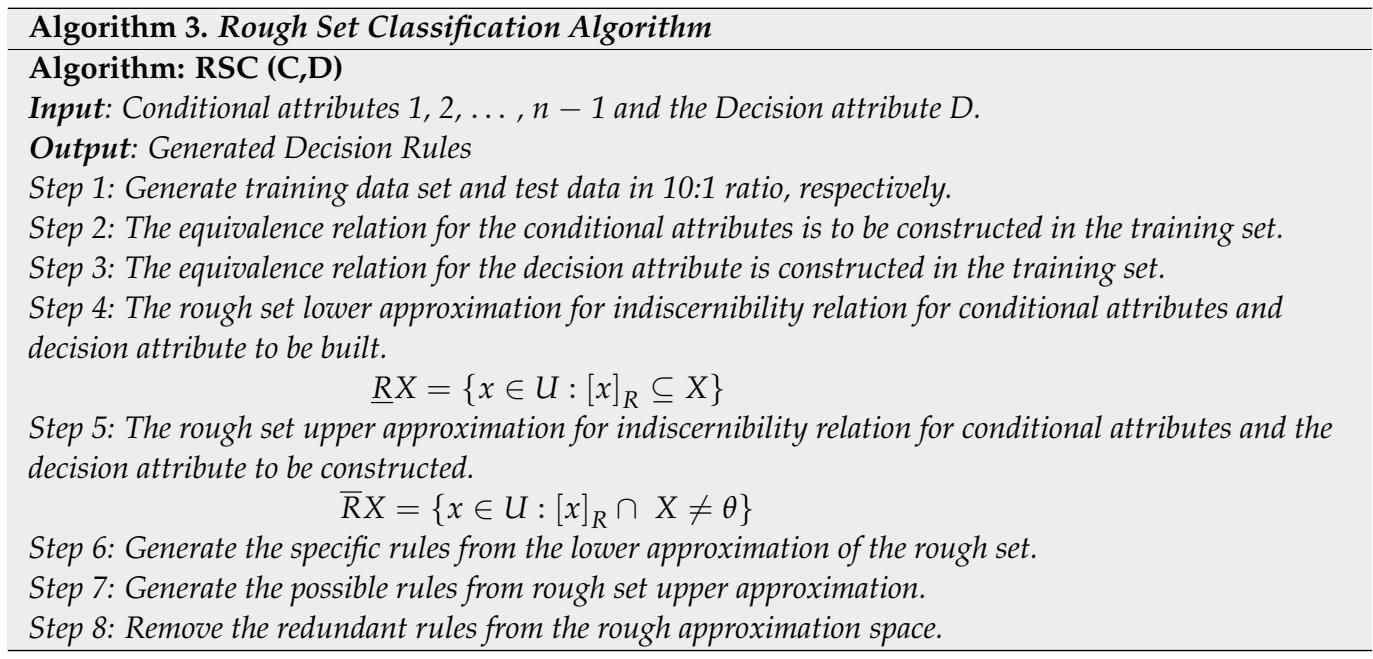

\section{Experimental Analysis}

In the proposed framework, the rough set algorithms analyze the peptides to predict anti-HIV-1 activity. Many features are reduced by selecting the significant feature subset by RSIHSQR and RSIHSRR algorithms. The feature subset is used as an input to the RSC algorithm, which classifies the peptides into two distinct classes as mentioned earlier, and the performance of the FS and the classification algorithms were analyzed with existing RSPSOQR and RSPSORR algorithms using Naïve Bayes, IBK, J48, Random Forest, and JRIP classifiers.

\subsection{Results}

The results of the feature selection algorithms are given in Table 2, and the outcome of classification algorithms are given in Table 3 .

\subsubsection{Performance Evaluation of Proposed FS Algorithms}

This study proposes using the rough set rapid reduct and relative reduct with better HS FS to reduce the dimensions of a given dataset. The proposed algorithms investigated the peptides collected from the HIPdb database. There are six physicochemical properties of the peptides, and 126 numerical features were generated, and the extracted features are subjected to the feature selection process to extract the best features. The proposed RSIHSQR and RSIHSRR algorithms are evaluated with RSPSOQR and RSPSORR algorithms. Table 2 explicitly illustrates the result of the proposed FS algorithms, which selects the 
minimum features of the existing algorithms. The classification algorithms evaluate the reliability of the feature subsets.

Table 2. Features Selected by FS algorithms.

\begin{tabular}{|c|c|c|}
\hline FS Algorithm & $\begin{array}{c}\text { No. of Selected } \\
\text { Features }\end{array}$ & Selected Features \\
\hline RSIHSRR & 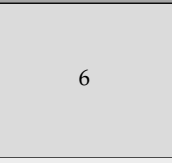 & $\begin{array}{l}\text { pk1_pk2_p1 } \\
\text { Hydrophobicity_mass_pk2_pI } \\
\text { Hydrophilicity_mass_pk2_pI } \\
\text { Hydrophobicity_hydrophilicity_mass_pk1_pk2 } \\
\text { Hydrophobicity_hydrophilicity_mass_pk2_pI } \\
\text { Hydrophilicity_mass_pk1_pk2_pI }\end{array}$ \\
\hline RSIHSQR & 7 & $\begin{array}{l}\text { pk2_p1 } \\
\text { Mass_pk1_pI } \\
\text { Hydrophobicity_hydrophilicity_pI } \\
\text { Hydrophobicity_hydrophilicity_pk1_pI } \\
\text { Hydrophobicity_hydrophilicity_mass_pk2_pI } \\
\text { Hydrophobicity_mass_pk2_pI } \\
\text { Hydrophilicity_mass_pk2_pI }\end{array}$ \\
\hline RSPSORR & 10 & $\begin{array}{l}\text { Hydrophobicity } \\
\text { Hydrophobicity_hydrophilicity } \\
\text { Hydrophilicity_pk1 } \\
\text { pk1_pI } \\
\text { pk2_pI } \\
\text { Hydrophobicity_hydrophilicity_pk1 } \\
\text { Hydrophobicity_pk1_pk2 } \\
\text { Hydrophobicity_pk1_pI } \\
\text { Hydrophobicity_hydrophilicity_mass_pk1_pk2 } \\
\text { Hydrophobicity_hydrophilicity_mass_pk1_pk2_pI }\end{array}$ \\
\hline RSPSOQR & 12 & $\begin{array}{l}\text { Hydrophobicity } \\
\text { pk2 } \\
\text { Hydrophobicity_mass } \\
\text { Hydrophobicity_pk1 } \\
\text { Hydrophilicity_mass } \\
\text { Mass_pI } \\
\text { pk1_pk2 } \\
\text { pk1_pI } \\
\text { Hydrophobicity_hydrophilicity_pk1 } \\
\text { Hydrophobicity_pk1_pk2 } \\
\text { Hydrophobicity_hydrophilicity_mass_pk1 } \\
\text { Hydrophobicity_hydrophilicity_mass_pk1_pk2 }\end{array}$ \\
\hline
\end{tabular}

Table 3. Results of the proposed framework and other FS and classification algorithms.

\begin{tabular}{|c|c|c|c|c|c|c|}
\hline $\begin{array}{l}\text { Classification } \\
\text { Technique }\end{array}$ & FS Algorithm & Precision & Recall & F-Measure & $\begin{array}{l}\text { Kulczynski } \\
\text { Index }\end{array}$ & $\begin{array}{c}\text { Fowlkes- } \\
\text { Mallows } \\
\text { Index }\end{array}$ \\
\hline \multirow{4}{*}{ Naïve Bayes } & RSIHSRR & 0.793 & 0.790 & 0.788 & 0.792 & 0.791 \\
\hline & RSIHSQR & 0.796 & 0.790 & 0.786 & 0.793 & 0.793 \\
\hline & RSPSORR & 0.625 & 0.593 & 0.578 & 0.609 & 0.609 \\
\hline & RSPSOQR & 0.620 & 0.593 & 0.581 & 0.607 & 0.606 \\
\hline \multirow{4}{*}{ IBK } & RSIHSRR & 0.964 & 0.958 & 0.952 & 0.961 & 0.961 \\
\hline & RSIHSQR & 0.927 & 0.926 & 0.926 & 0.927 & 0.926 \\
\hline & RSPSORR & 0.788 & 0.786 & 0.782 & 0.787 & 0.787 \\
\hline & RSPSOQR & 0.742 & 0.741 & 0.741 & 0.742 & 0.741 \\
\hline \multirow{4}{*}{ J48 } & RSIHSRR & 0.865 & 0.862 & 0.863 & 0.864 & 0.863 \\
\hline & RSIHSQR & 0.821 & 0.814 & 0.812 & 0.818 & 0.817 \\
\hline & RSPSORR & 0.679 & 0.677 & 0.677 & 0.678 & 0.678 \\
\hline & RSPSOQR & 0.783 & 0.783 & 0.783 & 0.783 & 0.783 \\
\hline \multirow{4}{*}{ Random Forest } & RSIHSRR & 0.823 & 0.823 & 0.823 & 0.823 & 0.823 \\
\hline & RSIHSQR & 0.823 & 0.823 & 0.823 & 0.823 & 0.823 \\
\hline & RSPSORR & 0.670 & 0.670 & 0.670 & 0.670 & 0.670 \\
\hline & RSPSOQR & 0.790 & 0.790 & 0.790 & 0.790 & 0.790 \\
\hline \multirow{4}{*}{ JRip } & RSIHSRR & 0.945 & 0.948 & 0.947 & 0.947 & 0.946 \\
\hline & RSIHSQR & 0.925 & 0.929 & 0.928 & 0.927 & 0.927 \\
\hline & RSPSORR & 0.827 & 0.826 & 0.824 & 0.827 & 0.826 \\
\hline & RSPSOQR & 0.838 & 0.832 & 0.830 & 0.835 & 0.835 \\
\hline \multirow{4}{*}{$\begin{array}{l}\text { Rough Set } \\
\text { Classifier } \\
\text { (RSC) }\end{array}$} & RSIHSRR & 0.956 & 0.942 & 0.941 & 0.948 & 0.940 \\
\hline & RSIHSQR & 0.915 & 0.924 & 0.938 & 0.968 & 0.962 \\
\hline & RSPSORR & 0.825 & 0.936 & 0.854 & 0.917 & 0.966 \\
\hline & RSPSOQR & 0.883 & 0.843 & 0.811 & 0.839 & 0.835 \\
\hline
\end{tabular}




\subsubsection{Assessment of FS with a Classification Algorithm}

Six classification algorithms assess the feature subsets selected by the four FS algorithms. The reliability of the FS algorithms is measured by Precision, Recall, F-measure, Kulczynski Index, and Fowlkes-Mallows Index [49,50]. These measures depend on True Positive (TP), False Positive (FP), and False Negative (FN).

$$
\begin{gathered}
\text { Precision }=\mathrm{TP} /(\mathrm{TP}+\mathrm{FP}) \\
\text { Recall }=\mathrm{TP} /(\mathrm{TP}+\mathrm{FN}) \\
\text { F-Measure }=2 *((\text { Precision } X \text { Recall }) /(\text { Precision }+ \text { Recall })) \\
\text { Kulczynski Index }=\frac{1}{2}(\text { Precision }+ \text { Recall }) \\
\text { Fowlkes-Mallows Index }=\sqrt{ }(\text { Precision * Recall })
\end{gathered}
$$

The results of the proposed framework of this study are evaluated using the performance measures (Equations (1)-(5)) of the 10-fold classification methods [51,52]. Table 3 shows the results of performance measures, in which many classifiers have revealed good results ( $>0.8$ ) with feature subset of proposed RSIHSQR and RSIHSRR feature selection algorithms. The validity measure greater than 0.8 represents the accuracy of the prediction rate, particularly with the peptide dataset [6].

\subsubsection{Assessment of RSC with Other Classification Algorithms}

Table 3 shows the performance of the RSC algorithm in the proposed framework compared with benchmark classifiers. It is believed that the rough set classifier has discovered better classification results of the anti-HIV-1 peptides and the RSC algorithm exposed high predictive accuracies with the proposed FS algorithms.

\section{Discussion}

Twenty-two different amino acids constitute numerous body proteins present in the human body. Of them, two are particular amino acids-selenocysteine and pyrrolysine, which were recently discovered. Each amino acid is unique in its own way and has specific characters that are responsible for its function. For example, histidine has a $\mathrm{pK}$ value of 6.1, which is close to the physiological $\mathrm{pH}$ of the blood, which makes it a critical blood buffer. It shields against changes in blood $\mathrm{pH}$ and maintains it within a narrow range of 7.38 to 7.42. The primary structure of proteins is formed by amino acids linked together by peptide bonds linearly. Some proteins also contain a circular primary structure, whereas some show branching. Hence, the functioning of the protein depends on its primary structure made of amino acids. Thus, in the same way, the functioning of an AHP is also, in turn, dependent on its amino acid composition. The amino acid may be linear or cyclic (aromatic), branched or unbranched, hydrophobic or hydrophilic, acidic or basic, and may also contain special groups with them.

This work on AHP focuses on understanding what essential attributes of an amino acid sequence determine if the AHP is effective against the virus. It is further focused on improving the efficiency in identifying the AHP and concurrently reduces the number of features needed to classify a peptide sequence to be AHP or not. This, in turn, makes the process easier in identifying AHP. The attributes chosen include:

Hydrophobicity: It is the property of an amino acid to present it as non-polar (chargeless) and, having no charges, they do not have any charge-to-charge communications that enable them to interface with water, and they dissolve in water very rarely; additionally, their substances can easily transverse the cell membrane made of the lipid bilayer. Almost all of the hydrophobic substances are essentially lipophilic.

Hydrophilicity: It is the property of the amino acid to present it as polar (charged); they are lipophobic and cannot transverse the cell membrane. 
Molecular Weight: It is calculated as the sum of the atomic weights of each element. For macromolecules such as proteins, methods based on viscosity and light-scattering can find molecular mass when crystallographic data are unavailable. Peptides of high molecular weight are less likely to be taken up by the cell compared to low molecular weight peptides.

pI (Isoelectric $\mathrm{pH}$ ): Amino acids have the unique property to exist as zwitterions. The $\mathrm{pH}$ at which the amino acid has no net charge (or an equal amount of positive and negative charges) is called $\mathrm{pK}$. The amino acid will have nil mobility in an electric field at this $\mathrm{pH}$. The solubility and buffering capacity will be minimum, whereas the perceptibility will be maximum.

pK1: When a particular amino acid is titrated with hydrochloric acid, the $\mathrm{pH}$ at which $50 \%$ of it exists as zwitterions and the other $50 \%$ as cations is called $\mathrm{pK} 1$.

pK2: The taken amino acid is titrated with Sodium Hydroxide. The $\mathrm{pH}$ at which 50\% of the molecules exist as zwitterions and the other $50 \%$ as anions is called pK2.

The proposed framework utilized these six attributes and proved maximum accuracy over existing FS and classification algorithms. The proposed RSIHSRR and RSC algorithms are observed as an appropriate framework for the accurate classification system with $96 \%$ in predicting anti-HIV-1 peptides.

\section{Conclusions}

HIV-1-fighting peptides have gained much attention in HIV / AIDS therapeutics. Researchers have adopted computational methods and algorithms in recent years to provide a cost-effective method in screening anti-HIV1 peptides, and feature selection is the vital pre-processing tool in peptide therapy. This study proposed a rough set-based FS technique, hybridized with population-based meta-heuristic algorithms, to classify the peptide sequences and solve dimensionality problems. Experimentation results show that the proposed framework discloses the most satisfactory solutions. It rapidly congregates in the problem space and finds the best reduct, which improves the predictive accuracy of the given dataset. The performance analysis report proved the effectiveness of RSIHSQR and RSIHSRR feature selection and Rough Set Classifier in analyzing peptide sequences to predict its activities.

As a future work, the proposed framework is recommended to any disease prediction analysis on peptide-related studies, such as viruses, bacteria, and microbes. The application of proposed framework can be extended by incorporating time complexity and deep learning algorithms.

Author Contributions: Conceptualization, A.T.A., B.M. and H.H.I.; methodology, B.M., H.H.I., A.T.A., N.A.K. and K.M.F.; software, B.M. and H.H.I.; validation, A.T.A., N.A.K., Y.J. and K.M.F.; formal analysis, B.M., A.T.A., H.H.I., N.A.K. and K.M.F.; investigation, J.L., H.H.I., A.T.A., N.A.K., K.M.F. and Y.J.; resources, J.L., B.M., N.A.K., K.M.F. and Y.J.; data curation, J.L., B.M. and Y.J.; writing-original draft preparation B.M., H.H.I., A.T.A., N.A.K., K.M.F. and J.L.; writing-review and editing B.M., H.H.I., A.T.A., N.A.K., K.M.F., Y.J. and J.L.; visualization, N.A.K., K.M.F. and Y.J.; supervision, H.H.I. and A.T.A. All authors have read and agreed to the published version of the manuscript.

Funding: The work is funded by Prince Sultan, Riyadh, Saudi Arabia.

Informed Consent Statement: Not applicable.

Data Availability Statement: The datasets underlying this article are accessible through the National Practitioner Data Bank portal [http://npdb-hipdb.com] accessed on 8 February 2022, antiviral peptides (AVPs) prediction web server [http://crdd.osdd.net/servers/avppred]. Accessed on 8 February 2022. 
Acknowledgments: The authors would like to acknowledge the support of Prince Sultan University for paying the Article Processing Charges (APC) of this publication. Special acknowledgement to Automated Systems \& Soft Computing Lab (ASSCL), Prince Sultan University, Riyadh, Saudi Arabia.

Conflicts of Interest: The authors declare no conflict of interest.

\section{References}

1. Niinomi, M. Titanium Alloys. In Encyclopedia of Biomedical Engineering; Elsevier: Amsterdam, The Netherlands, 2019; Volume 5, pp. 213-224. ISBN 9780128012383.

2. Tinguely, C.; Schild-Spycher, T.; Bahador, Z.; Gowland, P.; Stolz, M.; Niederhauser, C. Comparison of a conventional HIV $1 / 2$ line immunoassay with a rapid confirmatory HIV 1/2 assay. J. Virol. Methods 2014, 206, 1-4. [CrossRef] [PubMed]

3. Mehellou, Y.; Clercq, E.D. Twenty-six years of anti-HIV drug discovery: Where do we stand and where do we go? J. Med. Chem. 2021, 53, 521-538. [CrossRef]

4. Xiao, Y.F.; Jie, M.M.; Li, B.S.; Hu, C.J.; Xie, R.; Tang, B.; Yang, S.M. Peptide-Based Treatment: A Promising Cancer Therapy. J. Immunol. Res. 2015, 2015, 761820. [CrossRef]

5. Chertov, O.; Zhang, N.; Chen, X.; Oppenheim, J.J.; Lubkowski, J.; McGrath, C.; Ii, R.C.S.; Crise, B.J.; Malyguine, A.; Kutzler, M.A.; et al. Novel Peptides Based on HIV-1 gp120 Sequence with Homology to Chemokines Inhibit HIV Infection in Cell Culture. PLoS ONE 2011, 6, e14474. [CrossRef] [PubMed]

6. Poorinmohammad, N.; Mohabatkar, H. A Comparison of Different Machine Learning Algorithms for the Prediction of Anti-HIV-1 Peptides Based on Their Sequence-Related Properties. Int. J. Pept. Res. Ther. 2015, 21, 57-62. [CrossRef]

7. Iqbal, M.J.; Faye, I.; Samir, B.B.; Said, A.M. Efficient Feature Selection and Classification of Protein Sequence Data in Bioinformatics. Sci. World J. 2014, 2014, 173869. [CrossRef]

8. Al-Betar, M.; Khader, A.; Liao, I. A harmony search with multi-pitch adjusting rate for the university course timetabling. In Recent Advances in Harmony Search Algorithm; Geem, Z.W., Ed.; Springer: Berlin/Heidelberg, Germany, 2010; pp. 147-161.

9. Alia, O.M.; Mandava, R. The variants of the harmony search algorithm: An Overview. Artif. Intell. Rev. 2011, 36, 49-68. [CrossRef]

10. Zhu, Q.; Tang, X.; Elahi, A. Application of the novel harmony search optimization algorithm for DBSCAN clustering. Expert Syst. Appl. 2021, 178, 115054. [CrossRef]

11. Manjarres, D.; Landa-Torres, I.; Gil-Lopez, S.; Del Ser, J.; Bilbao, M.; Salcedo-Sanz, S.; Geem, Z.W. A survey on applications of the harmony search algorithm. Eng. Appl. Artif. Intell. 2013, 26, 1818-1831. [CrossRef]

12. Hasan, B.H.F.; Abu Doush, I.; Al Maghayreh, E.; Alkhateeb, F.; Hamdan, M. Hybridizing Harmony Search algorithm with different mutation operators for continuous problems. Appl. Math. Comput. 2014, 232, 1166-1182. [CrossRef]

13. Poursalehi, N.; Zolfaghari, A.; Minuchehr, A. Differential harmony search algorithm to optimize PWRs loading pattern. Nucl. Eng. Des. 2013, 257, 161-174. [CrossRef]

14. Yao, N.; Miao, D.; Pedrycz, W.; Zhang, H.; Zhang, Z. Causality measures and analysis: A rough set framework. Expert Syst. Appl. 2019, 136, 187-200. [CrossRef]

15. Inbarani, H.H.; Bagyamathi, M.; Azar, A.T. A Novel Hybrid Feature Selection Method Based on Rough Set and Improved Harmony Search. Neural Computing and Applications; Springer: Berlin/Heidelberg, Germany, 2015; Volume 26, pp. 1-22.

16. Mac Parthalain, N.; Jensen, R. Unsupervised fuzzy-rough set-based dimensionality reduction. Inf. Sci. 2013, 229, 106-121. [CrossRef]

17. Bagyamathi, M.; Inbarani, H.H. A Novel Hybridized Rough Set and Improved Harmony Search Based Feature Selection for Protein Sequence Classification. Big Data in Complex Systems: Challenges and Opportunities, Studies in Big Data; Springer: Berlin/Heidelberg, Germany, 2015; Volume 9, pp. 173-204.

18. Cao, Y.; Liu, S.; Zhang, L.; Qin, J.; Wang, J.; Tang, K. Prediction of protein structural class with Rough Sets. BMC Bioinform. 2006, 7, 20. [CrossRef]

19. Anand, A.; Pugalenthi, G.; Suganthan, P. Predicting protein structural class by SVM with class-wise optimized features and decision probabilities. J. Theor. Biol. 2008, 253, 375-380. [CrossRef]

20. Velayutham, C.; Thangavel, K. Unsupervised quick reduct algorithm using rough set theory. J. Electron. Sci. Technol. 2011, 9, 193-201.

21. Bagyamathi, M.; Azar, A.T.; Inbarani, H.H. Hybrid Rough Set with Black Hole Optimization Based Feature Selection Algorithm for Protein Structure Prediction. Int. J. Adv. Intell. Paradig. 2018, 10, 1. [CrossRef]

22. Bagyamathi, M.; Inbarani, H.H. Prediction of Protein Structural Classes by Pseudo Amino Acid Composition Using Improved Harmony Search Relative Reduct Feature Selection and Rough Set Classification Algorithms. Int. J. Invent. Comput. Sci. Eng. 2017, $4,55-65$.

23. Meher, P.K.; Sahu, T.K.; Saini V and Roa, A.R. Predicting anti-microbial peptides with improved accuracy by incorporating the compositional, physico-chemical and structural features into Chou's general PseAAC. Sci. Rep. 2017, 7, 42362. [CrossRef]

24. Azar, A.T.; Kumar, S.S.; Inbarani, H.H.; Hassanien, A.E. Pessimistic multi-granulation rough set-based classification for heart valve disease diagnosis. Int. J. Modeling Identif. Control. 2016, 26, 42-51. [CrossRef]

25. Zare, M.; Mohabatkar, H.; Faramarzi, F.K.; Beigi, M.M.; Behbahani, M. Using Chou's Pseudo Amino Acid Composition and Machine Learning Method to predict the Antiviral Peptides. Open Bioinform. J. 2015, 9, 13-19. [CrossRef] 
26. Bagyamathi, M.; Inbarani, H.H. Feature Selection using Improved Harmony Search Hybridized with Relative Reduct for Medical Data Classification. Int. J. Appl. Eng. Res. (IJAER) 2015, 10, 19476-19480.

27. Bagyamathi, M.; Inbarani, H.H. Feature Selection using Relative Reduct hybridized with Improved Harmony Search for Protein Sequence Classification. Int. J. Trend Res. Dev. 2015. Available online: http://www.ijtrd.com/papers/IJTRD1328.pdf (accessed on 10 January 2022).

28. Barrett, R.; Jiang, S.; White, A.D. Classifying antimicrobial and multifunctional peptides with Bayesian network models. Pept. Sci. 2018, 110, e24079. [CrossRef]

29. Tantisatirapong, S.; Davies, N.P.; Rodriguez, D.; Abernethy, L.; Auer, D.P.; Clark, C.A.; Arvanitis, T.N. Magnetic Resonance Texture Analysis: Optimal Feature Selection in Classifying Child Brain Tumors. In Proceedings of the XIII Mediterranean Conference on Medical and Biological Engineering and Computing, Seville, Spain, 25-28 September 2013; pp. 309-312.

30. Hajisharifi, Z.; Piryaiee, M.; Beigi, M.M.; Behbahani, M.; Mohabatkar, H. Predicting anticancer peptides with Chou's pseudo amino acid composition and investigating their mutagenicity via Ames test. J. Theor. Biol. 2014, 341, 34-40. [CrossRef]

31. Inbarani, H.H.; Azar, A.T.; Jothi, G. Supervised hybrid feature selection based on PSO and rough sets for medical diagnosis. Comput. Methods Programs Biomed. 2014, 113, 175-185. [CrossRef]

32. Azar, A.T. Neuro-fuzzy feature selection approach based on linguistic hedges for medical diagnosis. Int. J. Model. Identif Control. (IJMIC) 2014, 22, 195-206. [CrossRef]

33. Jothi, G.; Inbarani, H.H.; Azar, A.T. Hybrid tolerance-PSO based supervised feature selection for digital mammogram images. Int J. Fuzzy Syst Appl (IJFSA) 2013, 3, 15-30.

34. Qureshi, A.; Thakur, N.; Kumar, M. HIPdb: A database of experimentally validated HIV inhibiting peptides. PLoS ONE 2013, 8, e54908. [CrossRef]

35. Du, P.; Wang, X.; Xu, C.; Gao, Y. PseAAC-Builder: A Cross-Platform Stand-Alone Program for Generating Various Special Chou's Pseudo-Amino Acid Compositions. Anal. Biochem. 2012, 425, 117-119. [CrossRef]

36. Khosravian, M.; Faramarzi, F.K.; Beigi, M.M.; Behbahani, M.; Mohabatkar, H. Predicting Antibacterial Peptides by the Concept of Chou's Pseudo-Amino Acid Composition and Machine Learning Methods. Protein Pept. Lett. 2013, 20, 180-186. [CrossRef]

37. Beniwal, S.; Arora, J. Classification and feature selection techniques in data Mining. Int. J. Eng. Research Technol. 2012, 1, 2278-2284.

38. Kotsiantis, S.; Kanellopoulos, D. Discretization Techniques: A recent survey. GESTS Int. Trans. Comput. Sci. Eng. 2006, 32, 47-58.

39. Ali, R.; Siddiqi, M.H.; Lee, S. Rough set-based approaches for discretization: A compact review. Artif. Intell. Rev. 2015, 44, 235-263. [CrossRef]

40. Tsoukalas, A.; Parpas, P.; Rustem, B. A smoothing algorithm for finite min-max-min problems. Optim. Lett. 2008, 3, 49-62. [CrossRef]

41. Sathishkumar, E.N.; Thangavel, K.; Nishama, A. Comparative analysis of discretization methods for gene selection of breast cancer gene expression data. In Computational Intelligence, Cyber Security and Computational Models; Springer: Coimbatore, India, 2014; pp. 373-378.

42. Anaraki, J.R.; Eftekhari, M. Rough set based feature selection: A Review Fifth conference on information and knowledge technology (IKT). IEEE 2013, 2013, 301-306. [CrossRef]

43. Bagyamathi, M.; Inbarani, H.H. Prediction of Protein Structural Classes using Rough Set based Feature Selection and Classification Framework. J. Recent Res. Eng. Technol. 2017, 4, 1-9.

44. Geem, Z.W. Particle-Swarm Harmony Search for Water Network Design. Eng. Optim. 2009, 41, 297-311. [CrossRef]

45. Inbarani, H.H.; Banu, P.K.N.; Azar, A.T. Feature selection using swarm based relative reduct technique for fetal heart rate. Neural Comput. Appl. 2014, 25, 793-806. [CrossRef]

46. Kumar, S.U.; Inbarani, H.H.; Kumar, S.S. Improved Bijective-Soft-Set-Based Classification for Gene Expression Data. Computational Intelligence, Cyber Security and Computational Models. In Advances in Intelligent Systems and Computing; Springer: Berlin/Heidelberg, Germany, 2014; Volume 246, pp. 127-132.

47. Kumar, S.U.; Inbarani, H.H. PSO-based feature selection and neighborhood rough set-based classification for BCI multiclass motor imagery task. Neural Comput. Appl. 2016, 28, 3239-3258. [CrossRef]

48. Hall, M.; Frank, E.; Holmes, G.; Pfahringer, G.; Reutemann, P.; Witten, I.H. The WEKA data mining software: An update. ACM SIGKDD Explor. Newsl. 2009, 11, 10-18. [CrossRef]

49. Witten, I.H.; Frank, E.; Hall, M.A.; Pal, C.J. Data Mining: Practical Machine Learning Tools and Techniques; Morgan Kaufmann Publishers: Amsterdam, The Netherlands, 2016; ISBN 978-0-12-804291-5.

50. Thakur, N.; Qureshi, A.; Kumar, M. AVPpred: Collection and prediction of highly effective antiviral peptides. Nucleic Acids Res. 2012, 40, W199-W204. [CrossRef]

51. Salam, M.A.; Azar, A.T.; Elgendy, M.S.; Fouad, K.M. The Effect of Different Dimensionality Reduction Techniques on Machine Learning Overfitting Problem. Int. J. Adv. Comput. Sci. Appl. 2021, 12, 641-655. [CrossRef]

52. Azar, A.T.; Anter, A.M.; Fouad, K.M. Intelligent system for feature selection based on rough set and chaotic binary grey wolf optimization. Int. J. Comput. Appl. Technol. 2020, 63, 4-24. [CrossRef] 